\title{
IS THERE A PREDICTABLE CRITERION FOR MUTUAL SINGULARITY OF TWO PROBABILITY MEASURES ON A FILTERED SPACE?
}

\author{
W. Schachermayer and W. Schachinger \\ Department of Statistics, University of Vienna
}

\begin{abstract}
The theme of providing predictable criteria for absolute continuity and for mutual singularity of two density processes on a filtered probability space is extensively studied, e.g., in the monograph by J. Jacod and A. N. Shiryaev [JS]. While the issue of absolute continuity is settled there in full generality, for the issue of mutual singularity one technical difficulty remained open ([JS], p210): "We do not know whether it is possible to derive a predictable criterion (necessary and sufficient condition) for $P_{T}^{\prime} \perp P_{T}, \ldots$ ". It turns out that to this question raised in [JS] which we also chose as the title of this note, there are two answers: on the negative side we give an easy example, showing that in general the answer is no, even when we use a rather wide interpretation of the concept of "predictable criterion". The difficulty comes from the fact that the density process of a probability measure $P$ with respect to another measure $P^{\prime}$ may suddenly jump to zero.

On the positive side we can characterize the set, where $P^{\prime}$ becomes singular with respect to $P$ provided this does not happen in a sudden but rather in a continuous way - as the set where the Hellinger process diverges, which certainly is a "predictable criterion". This theorem extends results in the book of J. Jacod and A. N. Shiryaev [JS].
\end{abstract}

\section{INTRODUCTION}

We adopt the notation of [JS], which means that we are given two fixed probability measures $P, P^{\prime}$ on a filtered space $\left(\Omega,\left(\mathcal{F}_{t}\right)_{t \in \mathbb{R}^{+}}, \mathcal{F}\right)$ with right continuous filtration and $\mathcal{F}=\bigvee_{t} \mathcal{F}_{t}, Q=$ $\frac{P+P^{\prime}}{2}, z$ and $z^{\prime}$ denote the density processes of $P$ and $P^{\prime}$, relative to $Q$. We define the process $\left(Y_{t}\right)_{t \geq 0}$ by $Y_{t}=\sqrt{z_{t} z_{t}^{\prime}}$ and let $h=h\left(\frac{1}{2}\right)$ denote the Hellinger process of order $\frac{1}{2}$, i. e., the predictable increasing process $h$ such that $h_{0}=0$ and

$$
M=Y+Y_{-} \bullet h
$$

is a $Q$-martingale. For a stopping time $T$ we denote by $P_{T}$ and $P_{T}^{\prime}$ the restrictions of $P$ and $P^{\prime}$ to $\mathcal{F}_{T}$. We shall investigate the following question:

1.1 Problem. Under which conditions can we assert that $P_{T}^{\prime} \ll P_{T}$ or $P_{T}^{\prime} \perp P_{T}$ ? More generally, can we find a Hahn-decomposition of $\Omega$ into two sets, such that $P_{T}^{\prime}$ is absolutely continuous (resp. singular) with respect to $P_{T}$ on these sets?

The answer to this question should be in terms of a "predictable criterion". By this concept we mean that the answer should be in terms of the values of a predictable process, such as the Hellinger process $h$, evaluated at time $T$.

Before proceeding to answering this question we pause for some remarks: In order to avoid irrelevant complications at $t=0$ we suppose that $P_{0} \sim P_{0}^{\prime}$. We define the stopping time $S$ as the first moment when either $z$ or $z^{\prime}$ vanishes,

$$
S=\inf \left\{t: z_{t}=0 \text { or } z_{t}^{\prime}=0\right\} \text {. }
$$

Noting that (1) determines the Hellinger process $h$ only up to time $S$, we define $h$ to be constant after $S$, i. e., we consider the "Hellinger process in the strict sense" in the terminology of [JS]. We also introduce the Hellinger process $h(0)_{t}$ of order 0 as the compensator of the process $\mathbb{I}_{\llbracket S, \infty[\text {. }}$

Now we review the known results: there is a very satisfactory answer to our problem as regards the question of absolute continuity:

Key words and phrases. continuity and singularity of probability measures, Hellinger processes, stochastic integrals, stopping times.

Typeset by $\mathcal{A M N}_{\mathcal{M}}$-TEX 
1.2 Theorem. ([JS], Thm IV 2.6) In the above setting we have, for every stopping time $T$,

$$
P_{T}^{\prime} \ll P_{T} \Leftrightarrow P^{\prime}\left(G_{T}\right)=1,
$$

where $G_{T}=\left\{h_{T}<\infty\right\} \cap\left\{h(0)_{T}=0\right\}$.

Note that the set $G_{T}$ is defined in terms of the values of the two predictable processes $h$ and $h(0)$ at time $T$. To compare this result with the situation of mutual singularity below we make the following (trivial) reformulation: denote by $H$ the predictable, increasing, $[0, \infty]$-valued process $H_{t}=h_{t}+\infty \cdot h(0)_{t}($ where $\infty \cdot 0=0)$. Then

$$
P_{T}^{\prime} \ll P_{T} \Leftrightarrow P^{\prime}\left(H_{T}<\infty\right)=1
$$

For the question of mutual singularity (or, more generally, for the question of the Hahndecomposition) the situation is more subtle, the difficulty arising from the fact that $z_{t}$ may jump to zero as we shall presently see. Of course, we can always get rid of this difficulty by shifting it into the assumptions of a theorem: we thus obtain the subsequent result which directly follows from ([JS], lemma IV 2.12 a), b) and d)):

1.3 Theorem. In the above setting suppose in addition that

$$
P_{T}^{\prime}\left[S \leq T \text { and } z_{S-}>z_{S}=0\right]=0 .
$$

Then the restriction of $P_{T}^{\prime}$ to $\left\{h_{T}<\infty\right\}$ is absolutely continuous with respect to $P_{T}$ while the restriction of $P_{T}^{\prime}$ to $\left\{h_{T}=\infty\right\}$ is singular with respect to $P_{T}$.

In particular

$$
P_{T}^{\prime} \perp P_{T} \Leftrightarrow P^{\prime}\left(h_{T}=\infty\right)=1 .
$$

Our main task in this note is to analyze what we still can say when we don't use the simplifying assumption that $z_{t}$ is not allowed to jump to zero; unfortunately there is no hope for a complete analogue to theorem 1.2, as the following elementary example, which will be constructed in section 3 below, shows.

1.4 Example. There is a filtered space $\left(\Omega,\left(\mathcal{F}_{t}\right)_{t=0}^{2}, \mathcal{F}\right)$ equipped with two probability measures $P$ and $P^{\prime}$ with the following property:

There is no $[0, \infty]$-valued predictable process $H$ such that, for every stopping time $T$,

$$
P_{T}^{\prime} \perp P_{T} \Leftrightarrow P^{\prime}\left(H_{T}=\infty\right)=1 .
$$

In fact $\Omega$ may be chosen to consist only of 4 elements.

Despite this discouraging example we can formulate an interesting positive result, where we shift the problem, that $z_{t}$ may jump to zero from the assumption (as in theorem 1.3 above) into the conclusion of the theorem:

1.5 Theorem. Under the assumption that $P_{0} \sim P_{0}^{\prime}$ we have, for every stopping time $T$,

$$
\left\{S \leq T, z_{S_{-}}=0\right\}=\left\{h_{T}=\infty\right\} \quad P^{\prime}-\text { a.s. }
$$

The set on the left hand side may be interpreted as the set where $P_{T}^{\prime}$ is singular with respect to $P_{T}$, but in such a way that, as $t \nearrow S \leq T$, this singularity was not obtained by a "sudden jump", but rather in a continuous way. The assertion of the theorem is that - even in the presence of jumps of $z_{t}$ to zero - it is precisely this set which is characterized by the divergence of the Hellinger process.

Remark. We tried to formulate Thm 1.5 in a manner that suits best for comparison with the results in [JS], but there are other ways to state it. Actually, the assertion of Thm 1.5 is equivalent to the assertion

$$
\left\{z_{S_{-}}=0\right\}=\left\{h_{S}=\infty\right\} \quad P^{\prime}-\text { a.s. }
$$


To verify $(3) \Rightarrow\left(3^{\prime}\right)$, we take $T:=S$, and $\left(3^{\prime}\right) \Rightarrow(3)$ follows from

$$
\left\{S \leq T, z_{S_{-}}=0\right\}=\left\{S \leq T, h_{S}=\infty\right\}=\left\{S \leq T, h_{T}=\infty\right\}=\left\{h_{T}=\infty\right\} \quad P^{\prime}-\text { a.s. }
$$

where we used the constancy of $h$ from time $S$ on and the fact that $h_{T}<\infty$ for $T<S, P^{\prime}-$ a.s., which follows e.g. from ([JS], Thm IV 1.18).

Theorem 1.5 and example 1.4 answer the question raised in [JS] right after corollary IV 2.8 and also sharpen the assertions of ([JS], lemma IV 2.12). Our proof is quite different from the methodology used in [JS]: it uses a close monitoring of those paths of $z_{t}$, for which $z_{s_{-}}=0$, and an extension of the Borel-Cantelli lemma due to P. Lévy. Although the proof is elementary it is somewhat labourious and technical.

The paper is organized as follows: In section 2 we give the proof of theorem 1.5 and in section 3 we construct example 1.4 .

\section{Proof of theorem 1.5}

The inclusion $\left\{0<S \leq T, z_{S_{-}}=0\right\} \supseteq\left\{h_{T}=\infty\right\}, P^{\prime}$-a.s. is proved in ([JS], lemma IV 2.12a). The reverse inclusion can be deduced from ([JS], lemma IV $2.12 \mathrm{~b}+\mathrm{d}$ ) in the case, where $z$ isn't allowed to jump to 0 up to time $T$, i. e., under the assumption of theorem 1.3

$$
P_{T}^{\prime}\left[0<S \leq T, z_{S_{-}}>z_{S}=0\right]=0
$$

It remains to prove

$$
\left\{0<S \leq T, z_{S_{-}}=0\right\} \subseteq\left\{h_{T}=\infty\right\} \quad P^{\prime}-\text { a.s. }
$$

without assuming (4). As our proof will rely heavily on the fact that the local martingale $M$ given by (1) is a $Q$-martingale, we are aiming at $Q$-almost sure results. So the first thing to do is replace (5) by

$$
\left\{0<S \leq T, z_{S_{-}}=0 \text { or } z_{S_{-}}^{\prime}=0\right\} \subseteq\left\{h_{T}=\infty\right\} \quad Q-\text { a.s. }
$$

which is indeed equivalent to (5). We need the following lemma:

Lemma 2.1. Let $\left(A_{t}\right)_{t \in[0, \infty[}$ be an adapted increasing process on a filtered space $\left(\Omega,\left(\mathcal{F}_{t}\right), \mathcal{F}, \mathbb{P}\right)$, $\left(W_{n}\right)_{n \in \mathbb{N}}$ an increasing sequence of stopping times, such that for all $n \in \mathbb{N}$

$$
\mathbb{P}\left[W_{n-1}<\infty, \mathbb{P}\left[A_{W_{n}}-A_{W_{n-1}} \geq \alpha \mid \mathcal{F}_{W_{n-1}}\right]<\alpha\right] \leq 2^{-n}, \text { for some } \alpha>0
$$

Then

$$
\bigcap_{n=1}^{\infty}\left\{W_{n}<\infty\right\} \subseteq\left\{A_{\infty}=\infty\right\} \quad \mathbb{P}-\text { a.s. }
$$

Proof. Let $B_{n}=\left\{W_{n}<\infty\right\}, B=\bigcap_{n=1}^{\infty}\left\{W_{n}<\infty\right\}, E_{n}=\left\{A_{W_{n}}-A_{W_{n-1}} \geq \alpha\right\}$ and $\xi_{n}=$ $\mathbb{P}\left[E_{n} \mid \mathcal{F}_{W_{n-1}}\right]$. If $\mathbb{P}[B]=0,(7)$ is trivially satisfied, so we assume $\mathbb{P}[B]=a>0$. Since $B_{n} \searrow B$, we have

$$
\mathbb{P}\left[\xi_{n}<\alpha \mid B\right] \leq \frac{\mathbb{P}\left[\xi_{n}<\alpha, B_{n}\right]}{\operatorname{PP}[B]} \leq a^{-1} 2^{-n}
$$

The Borel-Cantelli lemma yields $\mathbb{P}\left[\xi_{n} \geq \alpha\right.$ i.o. $\left.\mid B\right]=1$, therefore

$$
B \subseteq\left\{\sum_{k=1}^{n} \xi_{k} \rightarrow \infty\right\} \quad \mathbb{P}-\text { a.s. }
$$


By Levy's extension of the Borel-Cantelli lemmas (cf. [S], p518 or [W], Thm 12.15)

$$
\left\{\sum_{k=1}^{\infty} \xi_{k}=\infty\right\}=\left\{\sum_{k=1}^{\infty} \mathbb{I}_{E_{k}}=\infty\right\} \quad \mathbb{P}-\text { a.s. }
$$

and the observation $A_{W_{n}} \geq \alpha \sum_{k=1}^{n} \mathbb{I}_{E_{k}}$ completes the proof.

Remark. Given $\alpha>0$ and a nonnegative random variable $X$, defined on a probability space $(\Omega, \mathcal{F}, \mathbb{P})$, we point out the following simple implications

$$
\mathbb{E}[X \wedge 2] \geq 3 \alpha \Rightarrow \mathbb{P}[X \wedge 2 \geq \alpha] \geq \alpha \Rightarrow \mathbb{E}[X \wedge 2] \geq \alpha^{2},
$$

which justify replacing hypothesis (6) by the equivalent hypothesis

$$
\mathbb{P}\left[W_{n-1}<\infty, \mathbb{E}\left[\left(A_{W_{n}}-A_{W_{n-1}}\right) \wedge 2 \mid \mathcal{F}_{W_{n-1}}\right]<\alpha^{\prime}\right] \leq 2^{-n}, \text { for some } \alpha^{\prime}>0
$$

We now proceed to the proof of theorem 1.5. First we define an increasing sequence of stopping times $\left(U_{n}\right)_{n \in \mathbb{N}}$ by

$$
U_{0}=0, \quad U_{n}=\inf \left\{t: U_{n-1} \leq t \leq T, z_{t} \neq 0, z_{t}^{\prime} \neq 0,\left\{\frac{z_{t}}{z_{U_{n-1}}}, \frac{z_{t}^{\prime}}{z_{U_{n-1}}^{\prime}}\right\} \not \subset\right] \frac{1}{2}, 2[\}, \text { for } n \geq 1
$$

where $\inf \emptyset:=\infty$. This definition ensures, that

$$
0 \leq Y_{\tau-} \leq 2 Y_{U_{n-1}} \quad Q \text {-a.s. for stopping times } \tau \in \rrbracket U_{n-1}, U_{n} \rrbracket
$$

and that on the set $\left\{U_{n}<\infty\right\}$ we have $\frac{z_{U_{n}}}{z_{U_{n-1}}} \leq \frac{1}{2}$ or $\frac{z_{U_{n}}}{z_{U_{n-1}}} \geq 2$ or $\frac{2-z_{U_{n}}}{2-z_{U_{n-1}}} \leq \frac{1}{2}$ or $\frac{2-z_{U_{n}}}{2-z_{U_{n-1}}} \geq 2$. Therefore

$$
z_{U_{n}} \in C\left(z_{U_{n-1}}\right) \quad Q \text {-a.s. on the set }\left\{U_{n}<\infty\right\} \text {, }
$$

where

$$
\left.C(x):=] 0, \frac{x}{2} \vee 2 x-2\right] \cup\left[2 x \wedge 1+\frac{x}{2}, 2[.\right.
$$

If $Q\left[\bigcap_{n=1}^{\infty}\left\{U_{n}<\infty\right\}\right]>0$, then for any $\mu_{0}>0$

$$
Q\left[z_{U_{n}} \in\right] 0, \mu_{0}[\cup] 2-\mu_{0}, 2\left[\mid U_{n}<\infty\right] \rightarrow 1, \quad \text { as } n \rightarrow \infty,
$$

since on the set $\bigcap_{n=1}^{\infty}\left\{U_{n}<\infty\right\}$ the sequence $\left(z_{U_{n}}\right)_{n \in \mathbb{N}}$ converges $Q$-a.s. to a random variable, which takes values in the two element set $\{0,2\}$ (other values are not possible by the definition of $\left.\left(U_{n}\right)_{n \in \mathbb{N}}\right)$, and since $Q\left[U_{n}<\infty\right] \rightarrow Q\left[\bigcap_{n=1}^{\infty}\left\{U_{n}<\infty\right\}\right]$ as $n \rightarrow \infty$. In particular, we have

$$
\left\{0<S \leq T, z_{S_{-}}=0 \text { or } z_{S_{-}}^{\prime}=0\right\}=\bigcap_{n=1}^{\infty}\left\{U_{n}<\infty\right\} \quad Q \text {-a.s. }
$$

so $\left(5^{\prime}\right)$ and thus theorem 1.5 will follow from lemma 2.1, if we can establish the truth of the subsequent lemma 2.2 .

Lemma 2.2. There exists $\gamma>0$ such that

$$
Q\left[U_{n-1}<\infty, \mathbb{E}\left[\left(h_{U_{n}}-h_{U_{n-1}}\right) \wedge 2 \mid \mathcal{F}_{U_{n-1}}\right]<\gamma^{2}\right] \rightarrow 0
$$

as $n \rightarrow \infty$.

Proof. Suppose to the contrary that, for any $0<\gamma<1$, there is a constant $\beta=\beta(\gamma)>0$ and for infinitely many $n$ (depending on $\gamma$ ) an $\mathcal{F}_{U_{n-1}}$-measurable set $E_{n-1}=E_{n-1}(\gamma) \subset\left\{U_{n-1}<\infty\right\}$, such that $Q\left[E_{n-1}\right] \geq \beta$ and on $E_{n-1}$ we have

$$
Q\left[h_{U_{n}}-h_{U_{n-1}} \geq \gamma \mid \mathcal{F}_{U_{n-1}}\right]<\gamma .
$$


For given small $\mu_{0}>0$ (to be chosen later), we can and will assume by passing again to a subsequence and invoking (10), that

$$
\left.z_{U_{n-1}} \in\right] 0, \mu_{0}[\cup] 2-\mu_{0}, 2[
$$

on an $\mathcal{F}_{U_{n-1}}$-measurable subset of $E_{n-1}$ of $Q$-probability at least $\frac{\beta}{2}$, which we again denote by $E_{n-1}$.

We then define a sequence of stopping times $\left(V_{n}\right)_{n \in \mathbb{N}}$ by

$$
V_{n}=\inf \left\{t: U_{n-1} \leq t, h_{t}-h_{U_{n-1}} \geq \gamma\right\},
$$

so that (11) implies

$$
\mathbb{E}\left[\mathbb{I}_{\left\{V_{n}<U_{n}\right\}} \mid \mathcal{F}_{U_{n-1}}\right]<\gamma \quad Q \text {-a.s. on } E_{n-1} \text {. }
$$

The definition of $\left(V_{n}\right)_{n \in \mathbb{N}}$ ensures, that

$$
h_{V_{n}}-h_{U_{n-1}}<2 \quad Q \text {-a.s. }
$$

since the jumps of $h$ are bounded by 1 (see, e. g. [JS], IV 1.30).

In order to get grip of $h$, we employ the Doob-Meyer decomposition of the supermartingale $Y$ given by (1)

$$
Y=M-Y_{-} \bullet h
$$

where $h$ is the Hellinger process of order $\frac{1}{2}$ in the strict sense and $M$ is a uniformly integrable martingale (cf. [JS], IV 1.18). Taking the difference $Y_{t}-Y_{U_{n-1}}$, and dividing by $Y_{U_{n-1}}$, we formally arrive at

$$
\frac{Y_{t}}{Y_{U_{n-1}}}=1+\frac{1}{Y_{U_{n-1}}}\left(M_{t}-M_{U_{n-1}}\right)-\left[\left(\frac{Y_{-}}{Y_{U_{n-1}}} \bullet h\right)_{t}-\left(\frac{Y_{-}}{Y_{U_{n-1}}} \bullet h\right)_{U_{n-1}}\right] \text {. }
$$

This can be looked upon as the Doob-Meyer decomposition of the supermartingale

$$
Y_{t}^{(n)}:=\mathbb{I}_{\left\{t \leq U_{n-1}\right\}}+\mathbb{I}_{\left\{t>U_{n-1}\right\}} \frac{Y_{t}}{Y_{U_{n-1}}}
$$

on the set $\left\{t>U_{n-1}\right\}$, which we rewrite as

$$
Y^{(n)}=M^{(n)}-Y_{-}^{(n)} \bullet h^{(n)},
$$

with $M_{t}^{(n)}:=1+\frac{1}{Y_{U_{n-1}}}\left(M_{t}-M_{U_{n-1}}\right) \mathbb{1}_{\left\{t>U_{n-1}\right\}}$ and $h_{t}^{(n)}:=\left(h_{t}-h_{U_{n-1}}\right) \mathbb{I}_{\left\{t>U_{n-1}\right\}}$. The martingale $M^{(n)}$ is again uniformly integrable and starts at 1 . In the sequel all expectations are taken with respect to $Q$. We are going to derive a contradiction in computing expectations at time $U_{n} \wedge V_{n}$ conditional on $\mathcal{F}_{U_{n-1}}$ :

$$
\mathbb{E}\left[Y_{U_{n} \wedge V_{n}}^{(n)} \mid \mathcal{F}_{U_{n-1}}\right]=\mathbb{E}\left[M_{U_{n} \wedge V_{n}}^{(n)} \mid \mathcal{F}_{U_{n-1}}\right]-\mathbb{E}\left[Y_{-}^{(n)} \bullet h_{U_{n} \wedge V_{n}}^{(n)} \mid \mathcal{F}_{U_{n-1}}\right]
$$

From corollary 2.1 below (which is a kind of "uniformly strict" Jensen inequality for the concave function $f(x)=\sqrt{x(2-x)}$ ) it follows that for properly chosen $\mu_{0}$ (cf. (12)) there exists $\epsilon>0$ such that on the set $E_{n-1}$ we have $Q$-a.s.

$$
\mathbb{E}\left[Y_{U_{n}}^{(n)} \mid \mathcal{F}_{U_{n-1}}\right] \leq 1-\epsilon+\epsilon Q\left[U_{n}=\infty \mid \mathcal{F}_{U_{n-1}}\right]
$$

This yields

$$
\begin{gathered}
\mathbb{E}\left[Y_{U_{n} \wedge V_{n}}^{(n)} \mid \mathcal{F}_{U_{n-1}}\right]=\mathbb{E}\left[Y_{U_{n}}^{(n)} \mid \mathcal{F}_{U_{n-1}}\right]-\mathbb{E}\left[Y_{U_{n}}^{(n)} \mathbb{I}_{\left\{V_{n}<U_{n}\right\}} \mid \mathcal{F}_{U_{n-1}}\right]+\mathbb{E}\left[Y_{V_{n}}^{(n)} \mathbb{I}_{\left\{V_{n}<U_{n}\right\}} \mid \mathcal{F}_{U_{n-1}}\right] \\
\leq\left[1-\epsilon+\epsilon Q\left[U_{n}=\infty \mid \mathcal{F}_{U_{n-1}}\right]\right]+2 \gamma
\end{gathered}
$$

where the estimate of the third term employs (8) and (13). 
Furthermore (8), (13) and (14) yield

$$
\begin{gathered}
\quad \mathbb{E}\left[\left(Y_{-}^{(n)} \bullet h\right)_{U_{n} \wedge V_{n}}-\left(Y_{-}^{(n)} \bullet h\right)_{U_{n-1}} \mid \mathcal{F}_{U_{n-1}}\right] \leq 2 \mathbb{E}\left[\left(h_{U_{n} \wedge V_{n}}-h_{U_{n-1}}\right) \mid \mathcal{F}_{U_{n-1}}\right] \\
=2 \mathbb{E}\left[\left(h_{V_{n}}-h_{U_{n-1}}\right) \mathbb{I}_{\left\{V_{n}<U_{n}\right\}} \mid \mathcal{F}_{U_{n-1}}\right]+2 \mathbb{E}\left[\left(h_{U_{n}}-h_{U_{n-1}}\right) \mathbb{I}_{\left\{V_{n} \geq U_{n}\right\}} \mid \mathcal{F}_{U_{n-1}}\right] \leq 4 \gamma+2 \gamma
\end{gathered}
$$

also $Q$-a.s. on the set $E_{n-1}$. Thus we obtain $Q$-a.s. on the set $E_{n-1}$

$$
1-\epsilon+\epsilon Q\left[U_{n}=\infty \mid \mathcal{F}_{U_{n-1}}\right] \geq 1-8 \gamma,
$$

which, if $\gamma$ is small enough $\left(\gamma=\frac{\epsilon}{10}\right.$ will do), can only be true for finitely many $n$, since $Q\left[U_{n}=\infty, U_{n-1}<\infty \mid \mathcal{F}_{U_{n-1}}\right] \rightarrow 0$ in $Q$-probability. This is the desired contradiction.

In order to have a means of discussing the problem of replacing $h$ in Theorem 1.5 by certain Hellinger-like processes (we will address this problem in more detail in the remark after corollary 2.1), we introduce some classes of concave functions, and prove a lemma, which is a "uniformly strict" version of Jensen's inequality for one of these classes of functions.

Definition 2.1. Let $\mathbb{E}_{0}$ be the class of concave functions $f:[0,2] \rightarrow \mathbb{R}^{+}$, satisfying $f(0)=0$ and

$$
1-\frac{a}{2}-2^{-b}>0, \quad 1+b-2^{a}>0
$$

where we denote

$$
a=\limsup _{x \rightarrow 0} \frac{x f^{\prime}(x)}{f(x)}, \quad b=\liminf _{x \rightarrow 0} \frac{x f^{\prime}(x)}{f(x)} .
$$

Let moreover $\mathbb{E}_{2}=\left\{f(\cdot): f(2-\cdot) \in \mathbb{E}_{0}\right\}$ and $\mathbb{E}=\mathbb{E}_{0} \cap \mathbb{E}_{2}$.

Note, that this classes are not empty. The set of admissible values of $(a, b)$ in $(15)$ is in fact a subset of $] 0,1\left[{ }^{2}\right.$ containing the set $\{(a, b): 0<a=b<1\}$. Practical members of $\mathbb{F}$ are the functions $f(x)=x^{\alpha}(2-x)^{\beta}$, where $0<\alpha, \beta<1$.

Lemma 2.3. Let $f \in \mathbb{F}_{0}$. Then there exist $\epsilon_{f}>0$ and $\mu_{f}>0$ such that for all $\left.\left.\mu \in\right] 0, \mu_{f}\right]$ the inequality

$$
\frac{\mathbb{E}[f(X)]}{f(\mu)} \leq 1-\epsilon_{f} p
$$

holds for any random variable $X$ ranging in $[0,2]$ and satisfying

$$
\mathbb{E} X=\mu, \quad \mathbb{P}[X \in D(\mu)]=p>0,
$$

where $D(\mu)=] 0,2[\backslash] \frac{\mu}{2}, 2 \mu[$.

Proof. Since $f$ is concave, the affine function $\ell(x)=f(\mu)+f^{\prime}(\mu)(x-\mu)$ satisfies $f(x) \leq \ell(x)$ and $f(\mu)=\ell(\mu)$. On the set $D(\mu)$ we even have $f(x) \leq \ell(x)-m(\mu)$ with

$$
m(\mu)=\min \left(\ell\left(\frac{\mu}{2}\right)-f\left(\frac{\mu}{2}\right), \ell(2 \mu)-f(2 \mu)\right) .
$$

Therefore

$$
\mathbb{E}[f(X)] \leq \mathbb{E}\left[\ell(X)-m(\mu) \mathbb{I}_{\{X \in D(\mu)\}}\right]=\ell(\mu)-m(\mu) \mathbb{P}[X \in D(\mu)]=f(\mu)-m(\mu) p .
$$

It remains to show that $\frac{m(\mu)}{f(\mu)}$ is bounded away from 0 uniformly as $\mu \rightarrow 0$ : By (16) we have $\mu f^{\prime}(\mu) \leq a f(\mu)(1+o(1))$ and $\frac{f^{\prime}(\mu)}{f(\mu)} \geq \frac{b}{\mu}(1+o(1))$. Integrating the latter inequality from $\frac{\mu}{2}$ to $\mu$ yields $\ln \frac{f(\mu)}{f\left(\frac{\mu}{2}\right)} \geq b \ln 2(1+o(1))$ and thus $f\left(\frac{\mu}{2}\right) \leq 2^{-b} f(\mu)(1+o(1))$. Therefore, as $\mu \rightarrow 0$,

$$
\ell\left(\frac{\mu}{2}\right)-f\left(\frac{\mu}{2}\right)=f(\mu)-\frac{\mu}{2} f^{\prime}(\mu)-f\left(\frac{\mu}{2}\right) \geq\left(1-\frac{a}{2}-2^{-b}+o(1)\right) f(\mu),
$$

and similarely $\ell(2 \mu)-f(2 \mu) \geq\left(1+b-2^{a}+o(1)\right) f(\mu)$. We have derived

$$
\frac{m(\mu)}{f(\mu)} \geq \min \left(1-\frac{a}{2}-2^{-b}, 1+b-2^{a}\right)+o(1) \text {. }
$$

Choosing now $\epsilon_{f}=\frac{1}{2} \min \left(1-\frac{a}{2}-2^{-b}, 1+b-2^{a}\right)$ and $\mu_{f}$ such, that the function $g(x)$ implied by the symbol $o(1)$ in the last inequality satisfies $|g(x)| \leq \epsilon_{f}$ for $\left.\left.x \in\right] 0, \mu_{f}\right]$ makes the proof complete. 
Corollary 2.1. There exist $\epsilon>0$ and $\mu_{0}>0$ such that for all $\left.\left.\mu \in\right] 0, \mu_{0}\right] \cup\left[2-\mu_{0}, 2[\right.$ and for any random variable $X$ ranging in $[0,2]$ and satisfying

$$
\mathbb{E} X=\mu, \quad \mathbb{P}[X \in C(\mu)]=p>0,
$$

where $C(\mu)$ is given by $(9)$, we have

$$
\mathbb{E}\left[\sqrt{\frac{X(2-X)}{\mu(2-\mu)}}\right] \leq 1-\epsilon p .
$$

Proof. This follows from Lemma 2.3 and the fact that $f(2-x)=f(x)$.

Remark. For given concave function $f$ we can define a supermartingale $Y_{t}=f\left(z_{t}\right)=M_{t}+A_{t}$, and ask, if the increasing process $H$, defined via $Y=M-Y_{-} \bullet H$ and required to be 0 at 0 and constant after $S$ (cf.(2)), can replace the Hellinger process $h$ of order $\frac{1}{2}$ in Theorem 1.5, i.e., satisfies

$$
\left\{Y_{S_{-}}=0\right\}=\left\{H_{S}=\infty\right\} \quad Q-\text { a.s. }
$$

for any $Q$-martingale $\left(z_{t}\right)_{t=0}^{\infty}$ with $z_{0}=1$ and $0 \leq z_{t} \leq 2$. Note that the Hellinger process $h\left(\frac{1}{2}\right)$ (resp. $h(\alpha)$, for $0<\alpha<1$ ) corresponds to the choice $f(z)=z^{\alpha}(2-z)^{1-\alpha}$.

One obvious condition, $f$ must satisfy, is $f(0)=f(2)=0$. Otherwise $H_{t}$, given by $\int_{0}^{t} \frac{d A_{s}}{Y_{s-}}$, would be finite $Q-$ a.s. on at least one of the sets $\left\{z_{s_{-}}=0\right\}$ resp. $\left\{z_{s_{-}}=2\right\}$.

Another necessary condition for $(17)$ is $f^{\prime}(0)=f^{\prime}(2)=\infty$. To see this, take $z_{t}=1+B_{t \wedge S}$, where $\left(B_{t}\right)$ is a standard Brownian motion with respect to $Q$ and $S=\inf \left\{t \geq 0:\left|B_{t}\right|=1\right\}$, and take $f(x)=1-|x-1|$, which satisfies $f^{\prime}(0)=1$. The Tanaka formula then reveals that $H_{t}=L_{t \wedge S}$, where $L$ denotes the local time at 0 of $\left(B_{t}\right)$. Now $S<\infty Q$-a.s. and therefore also $H_{S}<\infty Q$-a.s., but $Y_{S_{-}}=f\left(z_{S_{-}}\right)=0 Q$-a.s.

The methods of our paper suffice to prove the $\subseteq$-part of (17) for concave functions $f$ belonging to the class $\mathbb{F}$. In particular equations (8) and (14) remain true for $f \in \mathbb{F}$. The $\supseteq$-part of (17) for $f \in \mathbb{F}$ can be proved as in ([JS], lemma IV 2.12 a). Thus, in (17), $H$ can in particular be one of the Hellinger processes of order $\alpha$, for $0<\alpha<1$. However, there are concave functions satisfying $f(0)=f(2)=0$ and $f^{\prime}(0)=f^{\prime}(2)=\infty$, but not contained in $\mathbb{F}$, such as $f(x)=$ $x(2-x) \ln \left(\frac{x}{e}\right) \ln \left(\frac{2-x}{e}\right)$ and $f(x)=\left(\ln \left(\frac{x}{2 e}\right) \ln \left(\frac{2-x}{2 e}\right)\right)^{-1}$.

We do not know whether for general concave functions $f$ the conditions

$$
f(0)=f(2)=0, \quad f^{\prime}(0)=f^{\prime}(2)=\infty
$$

are also sufficient for (17) to hold, and leave this question for future research.

\section{Example 1.4}

Here we write down the example refered to in 1.4. Let $\left(\Omega,\left(\mathcal{F}_{t}\right)_{t=0}^{2}, \mathcal{F}\right)$ and two probability measures $P, P^{\prime}$ be given by

$$
\begin{aligned}
\Omega & =\left\{\omega_{1}, \omega_{2}, \omega_{3}, \omega_{4}\right\}, \\
\mathcal{F}_{0} & =\{\emptyset, \Omega\}, \\
\mathcal{F}_{1} & =\sigma\left(\left\{\omega_{1}\right\},\left\{\omega_{2}\right\},\left\{\omega_{3}, \omega_{4}\right\}\right), \\
\mathcal{F}_{2} & =\mathcal{F}=\sigma\left(\left\{\omega_{1}\right\},\left\{\omega_{2}\right\},\left\{\omega_{3}\right\},\left\{\omega_{4}\right\}\right), \\
P\left(\omega_{1}\right) & =2 P\left(\omega_{3}\right)=\frac{2}{3}, \\
P^{\prime}\left(\omega_{2}\right) & =2 P^{\prime}\left(\omega_{4}\right)=\frac{2}{3} .
\end{aligned}
$$

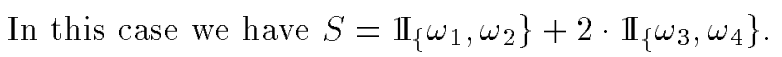


Claim. There is no $[0, \infty]$-valued predictable process $H$ such that, for every stopping time $T$,

$$
P_{T}^{\prime} \perp P_{T} \Leftrightarrow P^{\prime}\left(H_{T}=\infty\right)=1 .
$$

Proof. Assume that there is. Then $H_{1} \equiv$ const $<\infty, P^{\prime}$-a.s., since $\mathcal{F}_{0}$ is trivial and $P_{1}^{\prime} \not \perp P_{1}$. On the other hand $H_{S} \equiv \infty, P^{\prime}$-a.s., since $P_{S} \perp P_{S}^{\prime}$. Now on the set $\{S=1\}$, which has positive $P^{\prime}$-measure, we have conflicting definitions of $H_{1}$. This contradicts our assumption.

\section{Acknowledgement.}

The authors thank an anonymous referee for suggestions which led to considerable improvement of the presentation.

\section{REFERENCES}

[D] Durrett, Probability: Theory and Examples, Wadsworth, Inc., Belmont, California, 1991.

[DPK]. Dhaparidze, P. Spreij, E. Valkeila, On Hellinger Processes for Parametric Families of Experiments, preprint (1996)

[HW9. W. He, J. G. Wang, Remarks on Continuity, Contiguity and Convergence in Variation of Probability Measures, Séminaire de Probabilités XXII, Lecture Notes in Mathematics 1321 (1988), Springer, Berlin Heidelberg.

[J1] Jacod, Processus de Hellinger, absolue continuité, contiguité, Séminaire de Proba. de Rennes (1983), Rennes.

[J2] ـJacod, Théorèmes limite pour les processus, Ecole d'été de St-Flour XIII,1983, Lecture Notes in Mathematics 1117 (1985), Springer, Berlin Heidelberg.

[J3] Jacod, Filtered statistical models and Hellinger processes, Stochastic Processes and their Appl. 32 (1989), 3-45.

[J4]. Jacod, Convergence of flltered statistical models and Hellinger processes, Stochastic Processes and their Appl. 32 (1989), 47-68.

[JM]J. Jacod, J. Mémin, Caractéristiques locales et conditions de continuité absolue pour les semimartingales, Z. Wahrsch. Verw. Geb. 35 (1976), 1-37.

[JS] Jacod, A. N. Shiryaev, Limit Theorems for Stochastic Processes, Springer, Berlin, 1987.

[KLS] Kabanov, R. S. Liptser, A. N. Shiryaev, Absolute continuity and singularity of locally absolutely continuous probability distributions (Part I), Math. USSR Sbornik 35 (1979), 631-680; (Part II) 36 (1980), $31-58$.

[LS]F S. Liptser, A. N. Shiryaev, On the problem of "predictable" criteria of contiguity, Proc. 5th Japan-USSR Symp. Lecture Notes in Mathematics 1021 (1983), Springer, Berlin Heidelberg, 384-418.

[S] A. N. Shiryaev, Probability, Springer, New York, 1996.

[W] I. Williams, Probability with Martingales, Cambridge University Press, Cambridge, 1991. 